\title{
Are asymptomatic gastrointestinal findings on imaging more common in COVID-19 infection? Study to determine frequency of abdominal findings of COVID-19 infection in patients with and without abdominal symptoms and in patients with chest-only CT scans
}

\author{
Sree Harsha Tirumani ${ }^{1}$ - Ata A. Rahnemai-azar ${ }^{1}$ Jonathan D. Pierce ${ }^{1} \cdot K_{\text {Keval D. Parikh }}^{1} \cdot$ Sooyoung S. Martin ${ }^{1}$. \\ Robert Gilkeson ${ }^{1} \cdot$ Nikhil H. Ramaiya ${ }^{1}$
}

Received: 25 September 2020 / Revised: 11 December 2020 / Accepted: 14 December 2020 / Published online: 4 January 2021

(c) The Author(s), under exclusive licence to Springer Science+Business Media, LLC part of Springer Nature 2021

\begin{abstract}
Purpose To identify incidence of abdominal findings in COVID-19 patients with and without abdominal symptoms on various imaging modalities including chest-only CT scans and to correlate them with clinical, laboratory and chest CT findings. Materials and methods In this retrospective study, we searched our clinical database between March 1st, 2020 and May 22nd, 2020 to identify patients who had positive real-time reverse transcriptase polymerase chain reaction (RT-PCR) on throat swabs for COVID-19, had availability of clinical, laboratory information and had availability of CT scan of chest or abdominal radiograph, abdominal ultrasound or CT scan within 2 weeks of the diagnosis. Abdominal imaging findings on all imaging modalities were documented. Chest CT severity score (CT-SS) was assessed in all patients. Clinical and laboratory findings were recorded from the electronic medical record. Statistical analysis was performed to determine correlation of abdominal findings with CT-SS, clinical and laboratory findings.

Results Out of 264 patients with positive RT-PCR, 73 patients ( 38 males and 35 females; 35 African American) with mean age of 62.2 (range 21-94) years were included. The median CTSS was 13.5 (IQR 75-25 18-8). Most common finding in the abdomen on CT scans $(n=72)$ were in the gastrointestinal system in 13/72 patients (18.1\%) with fluid-filled colon without wall thickening or pericolonic stranding $(n=12)$ being the most common finding. Chest-only CT $(n=49)$ found bowel findings in 3 patients. CTSS did not differ in terms of age, sex, race or number of comorbidities but was associated with longer duration of hospitalization ( $p=0.0 .0256)$, longer intensive care unit stay $(p=0.0263)$, more frequent serum lactate dehydrogenase elevation $(p=0.0120)$ and serum C-reactive protein elevation $(p=0.0402)$. No statistically significant correlation of occurrence of bowel abnormalities with CTSS, clinical or laboratory features. Deep venous thrombosis was seen in 7/72 patients $(9.8 \%)$ with three patients developing pulmonary embolism

Conclusion Abnormal bowel is the most common finding in the abdomen in patients with COVID-19 infection, is often without abdominal symptoms and occurs independent of severity of pulmonary involvement, other clinical and laboratory features.
\end{abstract}

Keywords COVID-19 $\cdot$ Abdominal findings $\cdot$ CTSS $\cdot$ Bowel ischemia

\section{Introduction}

The novel severe acute respiratory syndrome coronavirus 2 (SARS-CoV-1) pandemic started in December 2019 and has resulted more than 32 million cases and nearly 1.0 million

Sree Harsha Tirumani

sreeharsha.tirumani@uhhospitals.org

1 Department of Radiology, University Hospitals Cleveland Medical Center, Case Western Reserve University, 11100 Euclid Ave, Cleveland, OH 44106, USA deaths word wide at the time of writing this article. In the USA, the number of cases has crossed 7 million with more than 200,000 fatalities. While the most common clinical presentation of symptomatic COVID-19 patients is upper 
respiratory tract infection including fever, shortness breath and cough [1], it is now known that COVID-19 is a multisystem disease with the virus targeting host cells expressing ACE-2 receptor including the airways, gastrointestinal system, liver and kidney $[2,3]$. It is also now known that significant number of patients have abdominal symptoms [4, 5]. In two recent meta-analysis by Parasa et al. and Cheung et al. $[6,7]$ the pooled rates for reporting diarrhea were $7.4 \%$ and $12.5 \%$, of nausea and vomiting were $4.6 \%$ and $10.2 \%$, respectively. The fecal positivity rate for COVID-19 was $40.5 \%$ and $48.1 \%$ respectively in these meta-analyses $[6,7]$. In a prospective study, high prevalence of GI symptoms was noted $(74 \%)$, though GI symptoms were prevalent $(50 \%)$ in COVID-19 negative patients as well [8].

Multiple studies have recently reported the abdominopelvic findings on CT scans of the abdomen and pelvis in COVID-19 patients with abdominal symptoms [9-18]. However, a significant number of COVID-19 patients undergo only a chest CT. While incidental detection of lung findings of COVID-19 on abdominal CT scans has been reported [19-21], the rate of positive abdominal findings on chest CT scans and their correlation with chest abnormalities has not been reported. The occurrence of incidental abdominal findings on CT scans in COVID-19 patients without abdominal symptoms is also not known. The purpose of this study was to identify incidence of abdominal findings in COVID-19 patients with and without abdominal symptoms at our hospital on various imaging modalities, especially on chest CT scans and to correlate them with clinical, laboratory and chest $\mathrm{CT}$ findings.

\section{Materials and methods}

\section{Patients}

This study was approved by the institutional review board with waiver for informed consent and was in compliance with the Health Insurance Portability and Accountability Act. We searched our clinical database of our single health care system with multiple hospitals between March 1st, 2020 and May 22nd, 2020 to identify patients who were tested with real-time reverse transcriptase polymerase chain reaction (RT-PCR) on throat swabs for COVID-19 and fulfilled the following inclusion criteria: 1-adult patients (age $>18$ years) with positive RT-PCR test for COVID-19, 2-availability of clinical, laboratory information 3-availability of CT scan of chest or abdominal radiograph, abdominal ultrasound or CT scan after the diagnosis, no later than 2 weeks after the positive RT-PCR test.

The demographics, clinical and laboratory characteristics of the patients were obtained by review of electronic medical records. Demographic and clinical information included age, sex, race, date of diagnosis, symptoms at the time of diagnosis, temperature, comorbidities, whether hospitalized and duration of hospitalization, whether admitted to intensive care unit (ICU) and duration of ICU stay, whether intubated, whether developed acute kidney injury during admission, specific treatment and whether deceased during the admission. Laboratory data on the date of the diagnosis or the CT scan of the chest or abdomen were recorded including serum glucose, potassium, sodium, urea creatinine, albumin, liver function tests, complete blood count, serum amylase, serum lipase, serum lactate dehydrogenase (LDH), C-Reactive Protein (CRP) and D-dimer.

\section{Image analysis}

All the available CT scans of the chest, abdomen and pelvis, radiographs of the abdomen, abdominal ultrasounds (right upper quadrant and renal) and venous Doppler ultrasound for deep venous thrombosis were reviewed by two radiologists in consensus ( $\mathrm{xx}$ and yy with 13 and 22 years of experience in radiology). Note was made of the number of all these exams. For CT scans, dates of the initial and follow-up scans (if available) and information about intravenous contrast administration was recorded. In patients with only chest CT scans, the findings in the upper abdomen were noted. Information about the presence of any prior cross-sectional imaging of the chest, abdomen and pelvis was also noted to compare findings seen on current imaging. All the images were reviewed on Picture Archiving Communication System (PACS; Sectra, Sweden). The radiologists were blinded to the radiology reports but were aware of positive RT-PCR test for COVID-19. Chest CT severity score (CT-SS) was assigned by one of the readers (yy). In patients with only abdominal CT scan, the CT-SS was derived by assessing the lung bases on CT scan and concurrent chest radiograph performed on the same day of the CT scan. The CT-SS was based on the scoring previously published by $\mathrm{Li}$ et al. [22]. A score of 0-5 was assigned to each lobe of the lung based on the extent of involvement of the lung $(0 \%,<5 \%, 5-25 \%$, $26-49 \%, 50-75 \%$ and $>75 \%$ for scores of $0,1,2,3,4$ and 5 , respectively) up to a maximum total score of 25 .

\section{Statistical analysis}

The most common abdominal findings were correlated with the CT-SS and with clinical and laboratory findings. Categorical variables were compared using Fisher's exact test or Chi-squared test and continuous variables were compared using Wilcoxon or Kruskal-Wallis test. For statistical analysis, a CT-SS score of 7 was used as cut off for categorizing patients into mild and severe/critical groups based on the study by Li et al. [22]. Furthermore, given that abnormal bowel was the most common finding 
(see section on results), comparison of various clinical and laboratory parameters was performed between those with abnormal bowel on $\mathrm{CT}$ and those without such finding. All $p$ values were based on a two-sided hypothesis. A $p$ value of $<0.05$ was considered to be statistically significant. All statistical analyses were conducted using JMP® Software (JMP® PRO, Version 14.0.0 SAS Institute Inc., Cary, NC, 1989-2007).

\section{Results}

264 contiguous patients were tested with real-time reverse transcriptase polymerase chain reaction (RT-PCR) on throat swabs for COVID-19. Of these, 108 patients had a positive RT-PCR test, of which 73 patients fulfilled the inclusion criteria. The demographic, clinical and laboratory features are shown in Table 1. Abdominal symptoms were present in $23 / 73$ patients $(31.5 \%)$ with diarrhea in $15(20.6 \%)$ patients.

\section{Image analysis}

The distribution of radiologic exams is listed in Table 2 .

\section{CT scan}

Of the 73 patients, only one patient did not have any form of CT performed during the diagnosis. The rest 72 patients had CT chest, CT abdomen or both. Analysis of the time interval between diagnosis and CT showed that chest CT $(n=64)$ was performed on the day or within 1 day of positive RT-PCR test in 48 patients, between 2 and 14 days of positive RT-PCR in 13 patients. One patient who had chest $\mathrm{CT}$ after more than 14 days (day 28) had a CT abdomen on day 11. In two patients, $C T$ chest was performed before positive RT-PCR test ( 5 days and 6 days, respectively). CT of the abdomen and pelvis $(n=24)$ was performed on the day of positive RT-PCR test in 17 patients and between 2 and 14 days of positive RTPCR in 6 patients. One patient had CT of the abdomen and pelvis 6 days before positive RT-PCR test. The indications for the $\mathrm{CT}$ of the abdomen and pelvis

Table 1 Demographic, clinical and laboratory features of the study cohort

\begin{tabular}{|c|c|}
\hline \multicolumn{2}{|l|}{ Parameter } \\
\hline Age (mean) & 62.2 (range $21-94$ ) years \\
\hline Sex & $\begin{array}{l}\text { Male-38 } \\
\text { Female-35 }\end{array}$ \\
\hline Race & $\begin{array}{l}\text { African American-35 } \\
\text { Caucasian-38 }\end{array}$ \\
\hline Death & 9 \\
\hline Comorbidities & $\begin{array}{l}\text { None }-10 \\
\text { One }-15 \\
\text { More than one }-48\end{array}$ \\
\hline Hospitalization in days & $\begin{array}{l}\text { None }-4 \\
1 \text { week or less-20 } \\
>1 \text { week }-49\end{array}$ \\
\hline Intensive care unit stay in days & $\begin{array}{l}\text { None }-33 \\
1 \text { week or less-14 } \\
>1 \text { week-26 }\end{array}$ \\
\hline Intubation & 21 \\
\hline Acute kidney injury & 28 \\
\hline Clinical features & $\begin{array}{l}\text { Upper respiratory symp- } \\
\text { toms-56 } \\
\text { Abdominal symptoms } \\
\text { (including diar- } \\
\text { rhea)-23 } \\
\text { Diarrhea-15 }\end{array}$ \\
\hline Temperature $\geq 38.0 \mathrm{C}$ & 10 \\
\hline White blood cell count $>11.3(n=80$, normal range $4.4-11.3)$ & 10 \\
\hline Neutrophils $\%>80(n=78$, normal range $40-80 \%)$ & 21 \\
\hline Lymphocyte $\%<13(n=78$, normal range $13-44 \%)$ & 22 \\
\hline Serum lactate dehydrogenase $>280(n=57$ patients, normal range $140-280 \mathrm{U} / \mathrm{L})$ & 29 \\
\hline C-reactive protein $>10(n=70$ patients, normal value $<10 \mathrm{mg} / \mathrm{dL})$ & 31 \\
\hline D dimer $>500(n=57$ patients, normal value $<500 \mathrm{ng} / \mathrm{mL}$ Fibrinogen equivalent units (FEU) $)$ & 46 \\
\hline
\end{tabular}


Table 2 List of exams performed in the study cohort

\begin{tabular}{|c|c|}
\hline Exam & Number of patients \\
\hline CT chest & 64 (with intravenous contrast-30) \\
\hline $\mathrm{CT}$ abdomen and pelvis & $\begin{array}{l}24 \text { (with intravenous contrast in 14, including one with both non- } \\
\text { contrast and contrast-enhanced) total } 27 \text { scans, } 3 \text { had more than } \\
\text { one scan }\end{array}$ \\
\hline Abdominal Radiographs & $\begin{array}{l}19 \text { (total-71, range } 1-12 \text { radiographs/patient) } \\
\text { (Indications: } 14-\text { tube check, rest for bowel obstruction versus ileus) }\end{array}$ \\
\hline Right upper quadrant ultrasound & $4($ total 4$)$ \\
\hline Renal ultrasound & $4($ total 4$)$ \\
\hline Venous ultrasound & $14($ total -15$)$ \\
\hline
\end{tabular}

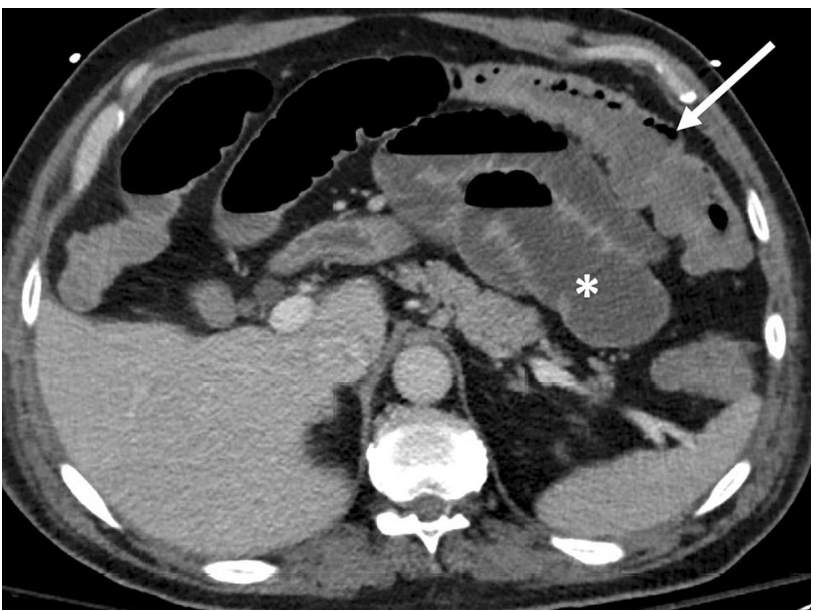

were abdominal pain $(n=14)$, diarrhea $(n=1)$, gastrointestinal bleeding $(n=1)$, urinary symptoms $(n=1)$, elevated liver function tests $(n=1)$ and trauma $(n=2)$. In the remaining 4 patients, the $\mathrm{CT}$ abdomen and pelvis was performed as part of CT chest without specific mention of the indication. Of the 72 patients, 43 patients had comparison CT or MRI in the PACS (36 patients within past 5 years).

Lung findings Of the 72 patients who had a CT performed during the diagnosis, there were positive findings in the lungs in 68 patients whereas four patients had normal lungs. In four of the 68 patients with positive lung findings, the lung abnormalities were first detected in lung bases on CT abdomen and pelvis. In 9 patients with no dedicated chest CT, the CT-SS was derived from the visualized portions of the lung and concurrent radiograph. The total CTSS was 7 or less in 17 patients and more than 7 in 55 patients. The median CTSS was 13.5 (IQR 75-25 18-8). The median CTSS for right lung was 7.5 (IQR 75-25 10.75-4) and for left lung was 6 (IQR 75-25 8-4).

Abdominal findings The most common finding in the abdomen on CT scans were in the gastrointestinal system including fluid-filled colon (defined as presence of homogeneous fluid attenuation contents in the lumen of colon without formed stool) without wall thickening or pericolonic stranding $(n=12)$ (Fig. 1), severe colitis with marked colonic wall thickening and pericolonic stranding $(n=1)$ (Fig. 2), gastritis $(n=2)$ and small bowel pneumatosis with portal venous gas $(n=1)$. Overall bowel abnormalities were noticed in $13 / 72$ patients $(18.1 \%)$.

Other findings included new fatty liver $(n=6)$, gallbladder sludge $(n=2)$, enlarged kidneys, $(n=1)$, bilateral renal infarcts $(n=1)$ and mesenteric stranding $(n=3)$.

Abdominal findings detected on chest-only CT scans 49 patients had only chest CT without abdominal CT of which 3 patients had a fluid-filled colon and one patient had fatty liver. Bowel abnormalities were not seen in any patient with abdominal symptoms who had only chest CT done.
Fig. 1 69-year-old male with COVID-19 pneumonia without abdominal symptoms. Axial contrast-enhanced CT images of the abdomen reveal fluid-filled colon (arrow) as well as fluid-filled, dilated small bowel (asterisk). CT severity score on chest CT was 15

\section{Abdominal radiographs}

Abdominal radiographs $(n=19)$ revealed diffuse gaseous dilation of small and large bowel consistent with ileus in five patients. The radiographs were normal in the remaining patients.

\section{Ultrasound}

Right upper quadrant ultrasound $(n=4)$ revealed gallbladder sludge in one patient, hepatitis in one patient, fatty liver in 2 patient and increased renal cortical echogenicity correlating with clinical picture of acute renal failure in one patient. Focused renal ultrasound $(n=4)$ revealed increased renal cortical echogenicity correlating with renal failure in one patient. The rest had either normal appearing kidneys or incidental findings like renal cysts. Doppler ultrasound of the extremities for venous thrombosis $(n=14)$ demonstrated deep venous thrombosis in 7 patients, of which three patients developed pulmonary thromboembolism. The venous 

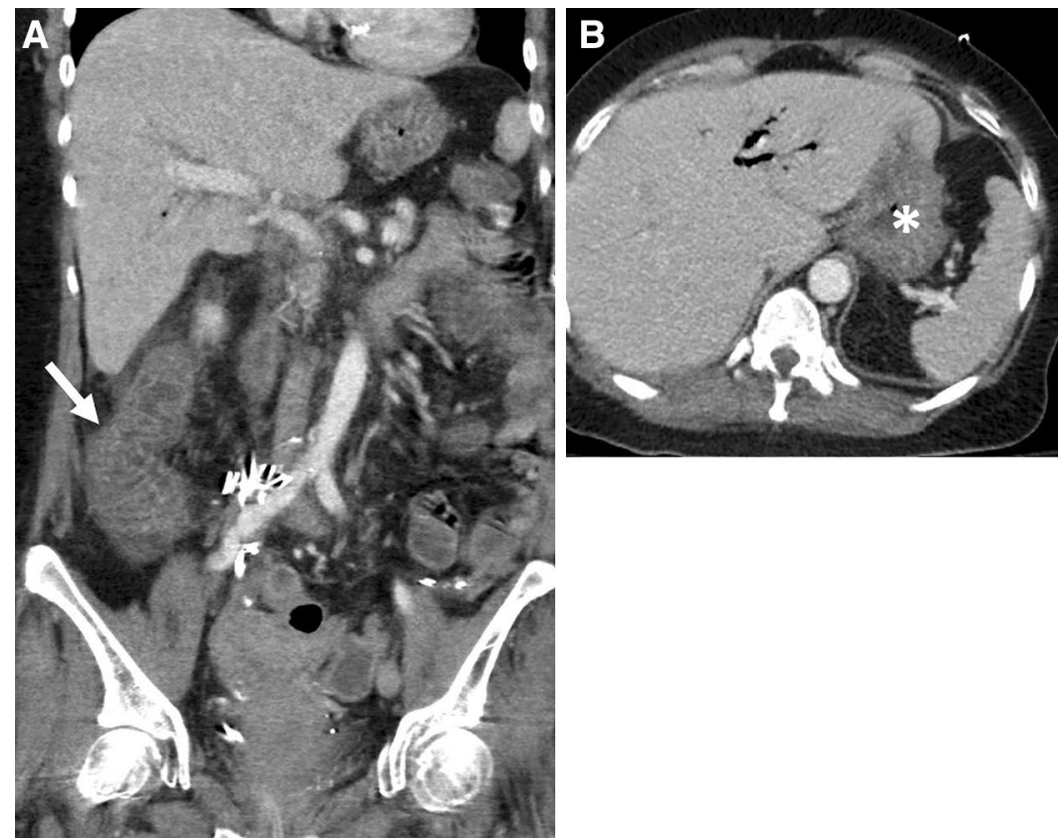

Fig. 2 53-year-old female with COVID-19 pneumonia with acute abdominal pain. Coronal (a) and axial $(\mathbf{b}, \mathbf{c})$ contrast-enhanced CT images of the abdomen reveal thickened and edematous gastric wall

thrombosis in one patient was secondary to May-Thurner syndrome which was managed with venous angioplasty.

\section{Correlation of CT-SS and bowel abnormalities with clinical and laboratory findings}

Patients in the severe/critical group (CTSS $>7$ ) did not differ from the mild group (CTSS $\leq 7)$ in terms of age, sex, race or number of comorbidities (Table 3). However, they had statistically significant longer duration of hospitalization $(p=\mathbf{0 . 0 2 5 6})$ and longer ICU stay $(p=\mathbf{0 . 0 2 5 6})$ than those in the mild group. There was no difference in the incidence of acute kidney injury between the two groups. Of the laboratory parameters, serum LDH and CRP were elevated more frequently in the severe/critical group than the mild group $(p=0.0120$ and 0.0402 , respectively). No statistically significant difference was noted in the rest of the parameters. There was no statistically significant difference between the two groups in terms of the occurrence of bowel abnormalities on CT scan, the most frequent imaging finding in the abdomen and pelvis. Patients with bowel abnormalities did not differ statistically from patients without bowel abnormalities in terms of clinical or laboratory features (Table 4). Patients with abnormal bowel had slightly lower temperature than those without abnormal bowel $(p=0.0152)$. Of the 13 patients with bowel abnormalities, 5 patients $(38.5 \%)$ had abdominal symptoms including diarrhea Of the 55 patients in the severe/critical group, 11 had bowel findings whereas (b, asterisk) and diffusely thickened and edematous colon with pericolonic stranding and fluid (a, c, arrow) consistent with gastritis and pancolitis. CT severity score on chest CT was 14

2 out of 17 patients in the mild group had bowel abnormalities, though this was not statistically significant ( $p=0.7195)$.

\section{Discussion}

In this study, abnormal bowel on CT was found in 13/72 patients (18.1\%) with COVID-19 infection of which the most frequent finding was fluid-filled colon in 12 patients $(16.7 \%)$ with severe colitis seen in one patient (1.4\%). Presence of bowel abnormalities was independent of clinical, laboratory and chest abnormalities in this study. The bowel finding was incidentally detected without abdominal symptoms in twothirds patients (8/13 patients, $61.5 \%)$. Bowel findings were noticed on chest CT in $6 \%$ patients (3/49patients with chest CT-only), none with abdominal symptoms.

The findings in our study are in partial agreement with two recently published studies $[12,13]$. Similar to the study by Goldberg-Stein et al. and Bhayana et al. [12, 13], the most common abdominal finding in our study was in the GI tract with fluid-filled colon being the most common finding. However, the incidence of bowel abnormalities was higher in the study by Bhayana et al. with abnormal bowel wall seen in $31 \%$ ( $18.1 \%$ in our study) and fluid-filled colon in $43 \%$ ( $16.7 \%$ in our study) [12]. In contrast to the findings of Bhayana et al. we did not find correlation of bowel findings with ICU admission[12]. Furthermore, only one patient in our series had pneumatosis suggestive of frank ischemia in 
Table 3 Correlation of CT severity score (CTSS) with clinical and laboratory parameters

\begin{tabular}{llll}
\hline & CTSS $</=7(n=17)$ & CTSS $>7(n=55)$ & $p$ value* \\
\hline Age (mean, range) years & $64(46-91)$ & $61.8(21-94)$ & 0.9102 \\
Sex ( male:female) & $9: 8$ & $28: 27$ & 1.0000 \\
Race (AA:C) & $9: 8$ & $25: 29$ & 0.7819 \\
Death & $2(12 \%)$ & $7(13 \%)$ & 1.0000 \\
Comorbidities (none or 1: $>1)$ & $7: 10$ & $19: 36$ & 0.7735 \\
Hospitalization in days (mean) & 8.1 & 15.5 & $\mathbf{0 . 0 2 5 6}$ \\
ICU stay in days (mean) & 2.4 & 8.3 & $\mathbf{0 . 0 2 6 3}$ \\
Intubation & $2(12 \%)$ & $19(35 \%)$ & 0.1231 \\
AKI & $4(24 \%)$ & $23(42 \%)$ & 0.2577 \\
WBC $>11.3$ & $3(18 \%)$ & $7(13 \%)$ & 0.6836 \\
Neutrophils $\%>80$ & $5(29 \%)$ & $16(29 \%)$ & 1.0000 \\
Lymphocyte $\%<13$ & $4(24 \%)$ & $17(31 \%)$ & 0.7588 \\
LDH $>280$ & $2(12 \%)$ & $26(47 \%)$ & $\mathbf{0 . 0 1 2 0}$ \\
CRP $>10$ & $4(24 \%)$ & $27(49 \%)$ & $\mathbf{0 . 0 4 0 2}$ \\
D dimer $>500$ & $10(59 \%)$ & $35(63 \%)$ & 1.0000 \\
Abdominal symptoms (yes) & $4(24 \%)$ & $19(35 \%)$ & 0.5541 \\
Abnormal bowel on CT & $2(12 \%)$ & $11(20 \%)$ & 0.7195 \\
Deep venous thrombosis & $2(12 \%)$ & $5(9 \%)$ & 0.6655 \\
Temperature (mean) & 37.2 & 37.2 & 0.9489 \\
\hline Valus & & &
\end{tabular}

Values in bold indicate statistically significant values

$A A: C$ African American:Caucasian, $I C U$ intensive care unit, $A K I$ acute kidney injury, $W B C$ white blood cell count, $L D H$ lactate dehydrogenase, $C R P$ C-reactive protein, CTSS CT severity score

*Fisher's exact test/Chi-squared test or Kruskal-Wallis test contrast to four patients in their study. ICU admission is frequently indicative of critical illness and often needs greater resuscitative efforts and greater risk of systemic hypoperfusion, greater chance of administration of systemic vasopressors all of which increased risk of bowel ischemia. The difference in incidence of bowel abnormalities between our study and the study by Bhayana et al. can be attributed to the incidence of ICU admission in the study by Bhayana et al. [12]. The presence of frank bowel ischemia in their study was attributed to COVID-19 associated coagulopathy. Patients with ICU admission are in general at risk of coagulopathy and the bowel findings could potentially be related to the systemic status rather than direct COVID-19 associated coagulopathy. Though in a recent study published form the same hospital, a higher rate of gastrointestinal complications were found in critically ill COVID-19 patients compared with propensity score-matched patients without COVID-19 [23], the results of this need validation in multiinstitutional studies. In the study by Goldberg-Stein et al., the incidence of bowel wall thickening was lower than the study by Bhayana et al. and comparable to our study [12, 13]. However, Goldberg-Stein et al. did not report the incidence of fluid-filled colon as their study was based on review of clinical reports rather than image re-review [13]. Another observation in this study was the low incidence of bowel findings in patients with chest-only $\mathrm{CT}$ which reassures the fact that chest-only CT does not miss many patients with bowel abnormalities, especially those without abdominal symptoms.

The results of our study are more reflective of pathogenesis and clinical picture of COVID-19 infection of the GI tract. Recent studies have shown that there is a high incidence of fecal positivity rate in COVID-19 infection (nearly $40-48 \%$ in two different meta-analyses) though the prevalence of GI symptoms is seen only in few patients (4-12\% in two meta-analyses) [6, 7]. The findings in our study are in strong agreement with these results in that two-thirds of patients with GI findings on imaging in our study were asymptomatic patients. Furthermore, the severity of COVID19 pulmonary manifestations or overall clinical or laboratory features did not determine occurrence of GI findings.

Similar to the GI findings, the overall incidence of abdominal findings especially biliary or gallbladder findings were also low in incidence in our study compared to other studies [12-14, 18]. This is similar to the observation of Barkmeier et al. who found that $63 \%$ of patients with abdominal symptoms and COVID-19 had negative CT scans [10]. The incidence of gallbladder sludge was $2 / 24$ patients with abdominal CT and 1/4 patients with ultrasound. While acute renal failure was diagnosed clinically in 38\% patients, positive findings of renal failure on ultrasound were noted in only one patient. However, these results are not comparable 
Table 4 Correlation of bowel findings with clinical and laboratory parameters

\begin{tabular}{llll}
\hline & $\begin{array}{l}\text { Bowel findings } \\
\text { absent }(n=59)\end{array}$ & $\begin{array}{l}\text { Bowel findings } \\
\text { present }(n=13)\end{array}$ & $p$ value* \\
\hline Age (mean, range) years & $61.0(21-94)$ & $67.6(49-91)$ & 0.2283 \\
Sex (male:female) & $31: 28$ & $6: 7$ & 07626 \\
Race (AA:C) & $29: 30$ & $6: 7$ & 1.0000 \\
Death & $6(10 \%)$ & $3(23 \%)$ & 0.1948 \\
Comorbidities $(>1)$ & $36(61 \%)$ & $11(85 \%)$ & 0.1183 \\
Hospitalization in days & 14.00 & 13.2 & 0.5716 \\
$\quad$ (mean) & & & \\
ICU stay in days (mean) & 7.0 & 6.4 & 0.5096 \\
Intubation & $16(27 \%)$ & $5(39 \%)$ & 0.5068 \\
AKI & $22(37 \%)$ & $6(46 \%)$ & 0.5496 \\
WBC $>11.3$ & $6(10 \%)$ & $4(31 \%)$ & 0.0737 \\
Neutrophils $\%>80$ & $15(25 \%)$ & $6(46 \%)$ & 0.1938 \\
Lymphocyte $\%<13$ & $17(29 \%)$ & $5(39 \%)$ & 0.7421 \\
Serum LDH $>280$ & $22(37 \%)$ & $7(54 \%)$ & 0.4880 \\
CRP $>10$ & $23(39 \%)$ & $8(62 \%)$ & 0.5339 \\
D dimer $>500$ & $36(61 \%)$ & $10(77 \%)$ & 0.5710 \\
Abdominal symptoms & $18(31 \%)$ & $5(39 \%)$ & 0.5320 \\
$\quad$ yes) & & & \\
CT SS $>7)$ & $44(75 \%)$ & $11(85 \%)$ & 0.7195 \\
Deep venous thrombosis & $6(10 \%)$ & $1(8 \%)$ & 1.0000 \\
Temperature (mean, $\left.{ }^{\circ} \mathrm{C}\right)$ & 37.3 & 36.9 & $\mathbf{0 . 0 1 5 2}$ \\
\hline Valu in & &
\end{tabular}

Value in bold indicates statistically significant value

$A A: C$ African American:Caucasian, $I C U$ intensive care unit, $A K I$ acute kidney injury, $W B C$ white blood cell count, $L D H$ lactate dehydrogenase, CRP C-reactive protein, CTSS CT severity score

*Fisher's exact test/Chi-squared test or Kruskal-Wallis test

to other studies as not all patients had ultrasound in our study. It is unclear if the occurrence of hepatobiliary (fatty liver, hepatitis, gallbladder sludge) and renal parenchymal changes are related to COVID-19 infection. Renal infarct was seen in one patient and has been reported in literature previously [11]. Deep venous thrombosis was present in nearly $9.6 \%$ of the patients in our study, higher than the study by Goldberg-Stein et al. (3.8\% in their study) [13] and O'Shea et al. (nearly $4 \%$ in their study) [24]. Importantly $43 \%$ patients with deep venous thrombosis (4\% overall) developed pulmonary embolism in our study. In the study by O'Shea et al. of the 308 patients with COVID-19, 21 patients developed pulmonary embolism and 13 patients had upper or lower extremity venous thrombosis [24]. In some recent studies the incidence of venous thromboembolism has been up to $30 \%$ [25-27].

There are several limitations to our study. This is a retrospective review of data collected form a single hospital. A significant number of patients in the study had chest-only $\mathrm{CT}$ and the findings in the abdomen were documented only from the upper abdomen. However, one of the goals of this study was to see how often we can find abdominal findings in patients with chest-only $\mathrm{CT}$ and whether these findings correlate with clinical features. The lack of fecal COVID-19 testing is another limitation. However, fecal COVID-19 testing is not the standard clinical practice currently. Whether some of the imaging findings are related to COVID-19 or not and if yes, the mechanisms involved behind them are unclear. Larger prospective studies with histopathologic correlation are needed to better understand the etiopathogenesis of these findings.

In conclusion, we found in our study that bowel abnormalities are the most common finding in the abdomen in patients with COVID-19 infection. We also found that bowel involvement is often asymptomatic and manifest as fluidfilled colon and occurs independent of severity of pulmonary involvement, other clinical and laboratory features. Larger studies are needed to validate the imaging findings in our study.

Funding Grant Support: None for the manuscript.

\section{Compliance with ethical standards}

Conflict of interest None of the other authors have any financial disclosures related to the content in the article.

\section{References}

1. Huang C, Wang Y, Li X et al (2020) Clinical features of patients infected with 2019 novel coronavirus in Wuhan, China. Lancet 395:497-506

2. Gu J, Han B, Wang J (2020) COVID-19: Gastrointestinal Manifestations and Potential Fecal-Oral Transmission. Gastroenterology 158:1518-1519

3. Qi F, Qian S, Zhang S, Zhang Z (2020) Single cell RNA sequencing of 13 human tissues identify cell types and receptors of human coronaviruses. Biochem Biophys Res Commun 526:135-140

4. Luo S, Zhang X, Xu H (2020) Don't Overlook Digestive Symptoms in Patients With 2019 Novel Coronavirus Disease (COVID19). Clin Gastroenterol Hepatol 18:1636-1637

5. Dietrich CG, Hubner D, Marx G, Bickenbach J, Bootsveld A (2020) Primary presentation of COVID-19 solely with gastrointestinal symptoms: a problem for the containment of the disease. Eur J Gastroenterol Hepatol. https://doi.org/10.1097/MEG.00000 00000001922

6. Cheung KS, Hung IFN, Chan PPY et al (2020) Gastrointestinal Manifestations of SARS-CoV-2 Infection and Virus Load in Fecal Samples From a Hong Kong Cohort: Systematic Review and Meta-analysis. Gastroenterology 159:81-95

7. Parasa S, Desai M, Thoguluva Chandrasekar V et al (2020) Prevalence of Gastrointestinal Symptoms and Fecal Viral Shedding in Patients With Coronavirus Disease 2019: A Systematic Review and Meta-analysis. JAMA Netw Open 3:e2011335

8. Chen A, Agarwal A, Ravindran N, To C, Zhang T, Thuluvath PJ (2020) Are Gastrointestinal Symptoms Specific for Coronavirus 2019 Infection? A Prospective Case-Control Study From the United States. Gastroenterology 159:1161-1163 e1162 
9. Abolyazid S, Alshareef S, Abdullah N, Khalil A, Hamza N, Salem A (2020) COVID-19 pneumonia identified by CT of the abdomen: A report of three emergency patients presenting with abdominal pain. Radiol Case Rep 15:2090-2094

10. Barkmeier DT, Stein EB, Bojicic K et al (2020) Abdominal CT in COVID-19 patients: incidence, indications, and findings. Abdom Radiol (NY). https://doi.org/10.1007/s00261-020-02747-5

11. Basara Akin I, Altay C, Eren Kutsoylu O, Secil M (2020) Possible radiologic renal signs of COVID-19. Abdom Radiol (NY). https ://doi.org/10.1007/s00261-020-02671-8

12. Bhayana R, Som A, Li MD et al (2020) Abdominal Imaging Findings in COVID-19: Preliminary Observations. Radiology. https:// doi.org/10.1148/radiol.2020201908:201908

13. Goldberg-Stein S, Fink A, Paroder V, Kobi M, Yee J, Chernyak V (2020) Abdominopelvic CT findings in patients with novel coronavirus disease 2019 (COVID-19). Abdom Radiol (NY) 45:2613-2623

14. Lei $P$, Zhang L, Han $P$ et al (2020) Liver injury in patients with COVID-19: clinical profiles, CT findings, the correlation of the severity with liver injury. Hepatol Int. https://doi.org/10.1007/ s12072-020-10087-1

15. Lui $\mathrm{K}$, Wilson MP, Low G (2020) Abdominal imaging findings in patients with SARS-CoV-2 infection: a scoping review. Abdom Radiol (NY). https://doi.org/10.1007/s00261-020-02739-5

16. Olson MC, Lubner MG, Menias CO et al (2020) RadioGraphics Update: Venous Thrombosis and Hypercoagulability in the Abdomen and Pelvis-Findings in COVID-19. Radiographics 40:E24-E28

17. Romero J, Valencia S, Guerrero A (2020) Acute Appendicitis During Coronavirus Disease 2019 (COVID-19): Changes in Clinical Presentation and CT Findings. J Am Coll Radiol 17:1011-1013

18. Shiralkar K, Chinapuvvula N, Ocazionez D (2020) Cross-Sectional Abdominal Imaging Findings in Patients With COVID-19. Cureus 12:e9538

19. Dane B, Brusca-Augello G, Kim D, Katz DS (2020) Unexpected Findings of Coronavirus Disease (COVID-19) at the Lung Bases on Abdominopelvic CT. AJR Am J Roentgenol 215:603-606
20. Hamilton NE, Adam GH, Ifan DL et al (2020) Diagnostic utility of additional whole-chest $\mathrm{CT}$ as part of an acute abdominal pain CT imaging pathway during the COVID-19 pandemic. Clin Radiol 75:592-598

21. King MJ, Lewis S, El Homsi M et al (2020) Lung base CT findings in COVID-19 adult patients presenting with acute abdominal complaints: case series from a major New York City health system. Eur Radiol. https://doi.org/10.1007/s00330-020-07040-z

22. Li K, Wu J, Wu F et al (2020) The Clinical and Chest CT Features Associated With Severe and Critical COVID-19 Pneumonia. Invest Radiol 55:327-331

23. El Moheb M, Naar L, Christensen MA et al (2020) Gastrointestinal Complications in Critically Ill Patients With and Without COVID-19. JAMA. https://doi.org/10.1001/jama.2020.19400

24. O'Shea A, Parakh A, Hedgire S, Lee SI (2020) Multisystem assessment of the imaging manifestations of coagulopathy in hospitalized patients with COVID-19. AJR Am J Roentgenol. https:// doi.org/10.2214/AJR.20.24132

25. Cui S, Chen S, Li X, Liu S, Wang F (2020) Prevalence of venous thromboembolism in patients with severe novel coronavirus pneumonia. J Thromb Haemost 18:1421-1424

26. Klok FA, Kruip M, van der Meer NJM et al (2020) Incidence of thrombotic complications in critically ill ICU patients with COVID-19. Thromb Res 191:145-147

27. Leonard-Lorant I, Delabranche X, Severac F et al (2020) Acute Pulmonary Embolism in Patients with COVID-19 at CT Angiography and Relationship to d-Dimer Levels. Radiology 296:E189-E191

Publisher's Note Springer Nature remains neutral with regard to jurisdictional claims in published maps and institutional affiliations. 